\title{
Pseudomonas lini Strain ZBG1 Revealed Carboxylic Acid Utilization and Copper Resistance Features Required for Adaptation to Vineyard Soil Environment: A Draft Genome Analysis
}

\author{
Kok-Gan Chan ${ }^{1 凶}$, Teik-Min Chong1, Tan-Guan-Sheng Adriann, Heng Leong Kher ${ }^{1}$, Catherine \\ Grandclément $^{2}$, Denis Faure ${ }^{2}$, Wai-Fong Yin¹, Yves Dessaux², Kar-Wai Hong ${ }^{1}$ \\ 1. Division of Genetics and Molecular Biology, Institute of Biological Sciences, Faculty of Science, University of Malaya, Kuala Lumpur, \\ Malaysia. \\ 2. Institute for Integrative Biology of the Cell (I2BC), CEA, CNRS, Université Paris-Sud, Université Paris-Saclay, 91198 Gif-sur-Yvette, \\ France.
}

$\triangle$ Corresponding author: kokgan@um.edu.my

(C) Ivyspring International Publisher. Reproduction is permitted for personal, noncommercial use, provided that the article is in whole, unmodified, and properly cited. See http://ivyspring.com/terms for terms and conditions.

Published: 2016.08 .05

\begin{abstract}
Pseudomonas lini strain ZBG 1 was isolated from the soil of vineyard in Zellenberg, France and the draft genome was reported in this study. Bioinformatics analyses of the genome revealed presence of genes encoding tartaric and malic acid utilization as well as copper resistance that correspond to the adaptation this strain in vineyard soil environment.
\end{abstract}

Key words: ZBG1

\section{Genome Announcement}

The first type strain of Pseudomonas lini which is CFBP 5737T, was isolated from Linum usitatissinum (flax) rhizosphere soil in 1993 at Dijon, France, attributing the name lini to this genus (1). In our study, $P$. lini strain ZBG1 was isolated from a vineyard soil in Zellenberg, France. In the databases, only one available genome sequence has been described for this species. Hence, acquiring the genome sequence of strain ZBG1 would be crucial to further comprehend the genomic information on this rarely reported bacterium as well as to elucidate the features of the strain for its adaptability in vineyard soil environments.

Bacterial genomic DNA was first extracted using Epicenter MasterPure DNA purification kit (Epicenter, Inc., USA) according to the manufacturer's protocol (2). Sequencing library was then prepared using Illumina Nextera DNA sample prep kit (Illumina, USA) followed by quantification using Qubit version 2.0 (Invitrogen, USA) (3). The library was sequenced using Illumina HiSeq 2500 (Illumina, USA). Subsequently, the quality of sequencing data generated was investigated using FastQC version 0.11.3, followed by trimming at Q20 and assembly using CLC Genomics Workbench version 7.0.4 $(4,5)$. Subsequently, ribosomal RNA (rRNA) sequence was predicted using RNAmmer version 1.2 and the predicted rRNA sequence was compared with NCBI BLAST database as well as EzTaxon database to verify the bacterial identity (6-8). The genomic sequence was then annotated using NCBI Prokaryotic Annotation Pipeline (PGAP) version 2.9.

A total of $7,611,538$ reads was generated and trimmed sequences were assembled into 327 contigs 
with an $N_{50}$ of $74,710 \mathrm{bp}$ and an average sequencing coverage at $64.5 \times$. The resulting draft genome size was at approximately $6.55 \mathrm{Mb}$ with a $\mathrm{G}+\mathrm{C}$ content of $58.7 \%$. The annotation pipeline showed that this genome consisted in 6,007 coding sequences, including 5,863 protein-coding genes, 4 rRNA-coding genes, 59 tRNA-coding genes, and 1 gene encoding other RNA. There were a total of 78 pseudogenes. The genome of $P$. lini ZBG1 contained several genes likely related to the potential of the bacteria to utilize several major organic acids of grapes (9-11), such as determinants for tartrate dehydrogenase (WP_050682071.1) and malate dehydrogenase (WP_007899225.1). Similar sequences of both tartrate dehydrogenase and malate dehydrogenase can be found in P. lini, Pseudomonas syringae, Pseudomonas fluorescens, Pseudomonas kilonensis, Pseudomonas chlororaphis, Pseudomonas marginalis, Pseudomonas corrugata and Pseudomonas mandelii $(12,13)$ which were isolated from grape and/or vineyard-related environment.

In most vineyards, copper sulfate has been widely applied as a fungicide $(14,15)$. Consequently, a series of copper resistance genes encoding copper chaperones (WP_038978765.1 and WP_008069687.1), copper oxidases CopA (WP_050682449.1) CopB (WP_050682450.1), CopC (WP_038980050.1), CopD (WP_050683399.1 and WP_050682451.1) have been identified in the genome. The CopC periplasmic protein and the CopD membrane protein jointly participated in the copper trafficking by delivering essential copper through the inner membrane into the cytoplasm $(16,17)$. The CopA and CopB proteins sequester the excess essential copper (16). Such observations demonstrated the adaptability of this bacterium towards vineyard soil environment.

By comparison with the genome of $P$. lini strain DSM 16768, both genomes were differed by $49,576 \mathrm{bp}$, in which the genome size of strain ZBG1 is slightly bigger. The total annotated amino acid sequences were also compared using OrthoVenn (18). Despite sharing a total of 5,221 ortholog clusters, 13 unique ortholog clusters were identified within both genomes of $P$. lini strain ZBG1 and strain DSM 16768, respectively (Figure 1 ).

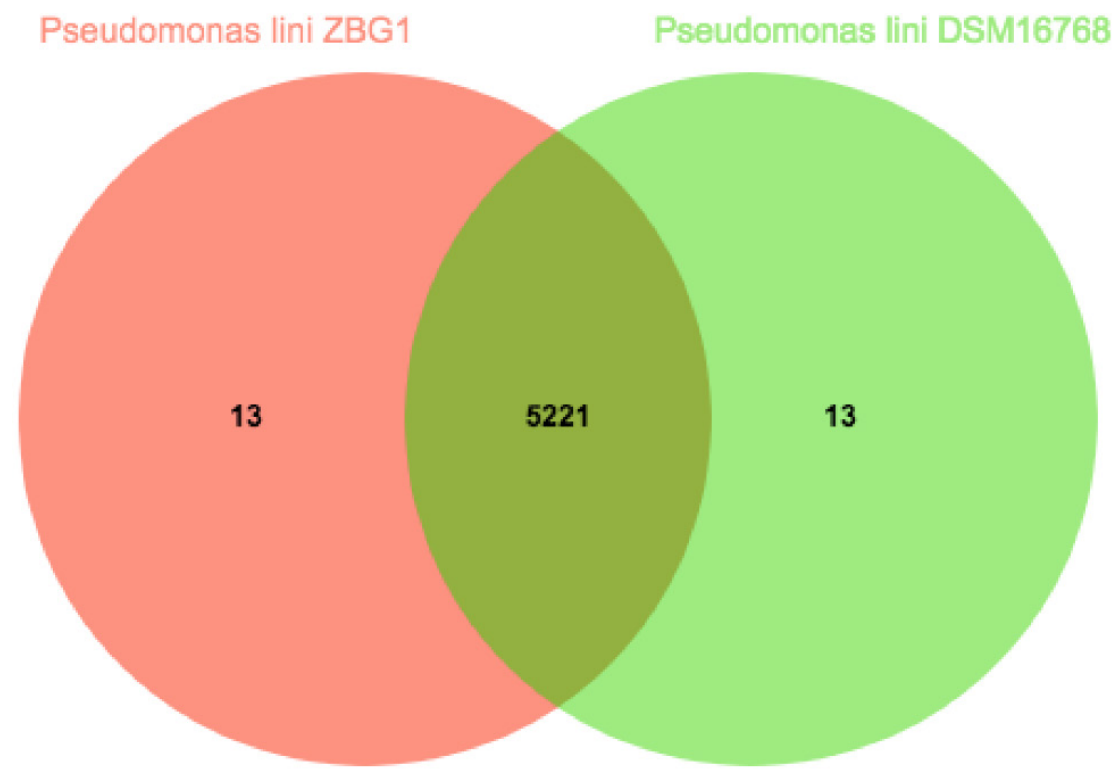

Figure 1. Comparison of both P. lini ZBG1 and DSM 16867. Both genomes shared a total of 5,221 ortholog clusters.

\section{Nucleotide sequence accession numbers.}

This draft-genome shotgun project has been deposited at DDBJ/EMBL/GenBank under the accession number LFQO00000000. The version described in this paper is the first version, LFQO00000000.

\section{Acknowledgement}

K.-G.C., T.-M.C., K.-W.H., and Y.D. thank the French Embassy in Kuala Lumpur (Malaysia) for the French Fellowships. This research was supported by the University of Malaya through the HIR Grants (UM-MOHE HIR Grant UM C/625/1/HIR/MOHE/ CHAN/14/1, no. H-50001-A000027; UM-MOHE HIR Grant UM
C/625/1/HIR/MOHE/CHAN/01, 
no. A000001-50001) awarded to K.-G.C., which are gratefully acknowledged.

\section{Competing Interests}

The authors have declared that no competing interest exists.

\section{References}

1. Delorme S, Lemanceau P, Christen R, et al. Pseudomonas lini sp. nov., a novel species from bulk and rhizospheric soils. International Journal of Systematic and Evolutionary Microbiology, 2002. 52(2): 513-523.

2. Tan W-S, Yin W-F, and Chan K-G. Insights into the quorum-sensing activity in Aeromonas hydrophila strain $\mathrm{m} 013$ as revealed by whole-genome sequencing. Genome Announcements, 2015. 3(1): e01372-14.

3. Chan K-G, Sulaiman J, Yong DA, et al. Draft genome perspective of Staphylococcus saprophyticus strain SU8, an N-acyl homoserine lactone-degrading bacterium. Genome Announcements, 2015. 3(5): e01097-15.

4. Mohamad NI, Tan W-S, Chang C-Y, et al. Analysis of quorum-sensing Pantoea stewartii Strain M073A through whole-genome sequencing. Genome Announcements, 2015. 3(1): e00022-15.

5. Andrews S. FastQC: A quality control tool for high throughput sequence data. Reference Source, 2010.

6. Lim Y-L, Ee R, How K-Y, et al. Complete genome sequencing of Pandoraea pnomenusa RB38 and Molecular Characterization of Its $\mathrm{N}$-acyl homoserine lactone synthase gene ppnI. PeerJ, 2015. 3: e1225.

7. Chun J, Lee J-H, Jung Y, et al. EzTaxon: a web-based tool for the identification of prokaryotes based on $16 \mathrm{~S}$ ribosomal RNA gene sequences. International Journal of Systematic and Evolutionary Microbiology, 2007. 57(10): 2259-2261.

8. Lagesen K, Hallin P, Rødland EA, et al. RNAmmer: consistent and rapid annotation of ribosomal RNA genes. Nucleic Acids Research, 2007. 35(9): 3100-3108.

9. Iland P and Coombe B. Malate, tartrate, potassium, and sodium in flesh and skin of Shiraz grapes during ripening: concentration and compartmentation. American Journal of Enology and Viticulture, 1988. 39(1): 71-76.

10. Kohn LD and Jakoby WB. Tartaric Acid Metabolism IV. Crystalline L-malic dehydrogenase from Pseudomonas acidovorans. Journal of Biological Chemistry, 1968. 243(10): 2472-2478.

11. Lonvaud-Funel A and De Saad AS. Purification and properties of a malolactic enzyme from a strain of Leuconostoc mesenteroides isolated from grapes. Applied and Environmental Microbiology, 1982. 43(2): 357-361.

12. Leveau J and Tech J. Grapevine microbiomics: bacterial diversity on grape leaves and berries revealed by high-throughput sequence analysis of $16 \mathrm{~S}$ rRNA amplicons. in International Symposium on Biological Control of Postharvest Diseases: Challenges and Opportunities 905. 2010.

13. Surico G, Bandinelli R, Braccini P, et al. On the Factors that May Have Influenced the Esca Epidemic in the Eighties in Tuscany. Phytopathologia Mediterranea, 2004. 43(1): 136-143.

14. Johnson GF. The early history of copper fungicides. Agricultural History, 1935. 9(2): 67-79.

15. Fernández-Calviño D, Soler-Rovira P, Polo A, et al. Enzyme activities in vineyard soils long-term treated with copper-based fungicides. Soil Biology and Biochemistry, 2010. 42(12): 2119-2127.

16. Arnesano F, Banci L, Bertini I, et al. Solution structure of CopC: a cupredoxin-like protein involved in copper homeostasis. Structure, 2002. 10(10): 1337-1347.

17. Bondarczuk K and Piotrowska-Seget Z. Molecular basis of active copper resistance mechanisms in Gram-negative bacteria. Cell Biology and Toxicology, 2013. 29(6): 397-405.

18. Wang Y, Coleman-Derr D, Chen G, et al. OrthoVenn: a web server for genome wide comparison and annotation of orthologous clusters across multiple species. Nucleic Acids Research, 2015. 43(W1): W78-W84. 